EPJ Web of Conferences 66, 01012 (2014)

DOI: 10.1051 epjconf/ 20146601012

(C) Owned by the authors, published by EDP Sciences, 2014

\title{
Nuclear lattice simulations: Status and perspectives ${ }^{\star}$
}

\author{
Ulf-G. Meißner ${ }^{1,2, a}$ \\ ${ }^{1}$ Universität Bonn, HISKP \& BCTP, D-53115 Bonn, Germany \\ ${ }^{2}$ Forschungszentrum Jülich, IAS, IKP \& JCHP, D-52425 Jülich, Germany
}

\begin{abstract}
I review the present status of nuclear lattice simulations. This talk is dedicated to the memory of Gerald E. Brown.
\end{abstract}

\section{Prologue: In memoriam of Gerald E. Brown}

Just a few days before this talk, my beloved teacher Gerry Brown passed away in the age of 86 . Not only me but also the nuclear physics community owes him a lot. We will always remember him as a superb physicist, a gifted teacher and a wonderful (hu)man. Thank you, Gerry!

\section{Introduction}

This talk is a natural extension of my presentation at INPC 2004, where I gave a plenary talk titled "Modern theory of nuclear forces" [1]. There, I presented the developments of the chiral effective field theory (EFT) approach to the nuclear force problem initiated by Steven Weinberg in 1990 [2]. This scheme allows for a consistent and accurate description of the forces between two, three and four nucleons, based on the symmetries of QCD, with controllable theoretical errors and a systematic scheme to improve the precision. Much progress has been made in the applications and tests of these forces in few-nucleon systems, for a cornucopia of bound state or scattering calculations, see e.g. the recent reviews [3, 4] and also the talk by Machleidt at this conference. It is therefore natural to ask how to address the properties of nuclei with $A>4$. There are essentially two ways of doing that. On the one hand, one can combine standard many-body techniques like the (no-core) shell model or coupled cluster approaches with these forces as discussed e.g by Bacca [5] and Roth [6] (often using a low-momentum a.k.a. soft representation of the forces). On the other hand, one can try to devise a new approach to tackle the many-body problem that is tailored to the chiral EFT approach. This novel scheme, that combines the EFT description of the few-nucleon forces with Monte-Carlo simulation techniques, will be discussed here. It is called nuclear lattice simulations and was introduced for atomic nuclei in Ref. [7] and subsequently developed in few-nucleon systems (see the review by Lee that contains references to earlier related and pioneering work [8]). Here, I will discuss the progress made in the description of nuclei that has been made in the last years, particularly with respect to the spectrum and structure of ${ }^{12} \mathrm{C}$ and other $\alpha$-cluster type nuclei.

\footnotetext{
${ }^{\star}$ Lecture sponsored by The European Physical Journal A - Hadrons and Nuclei

ae-mail: meissner@hiskp.uni-bonn.de
} 


\section{Chiral effective field theory on a lattice}

The starting point of chiral EFT for nuclei is the standard effective pion-nucleon Lagrangian supplemented by multi-nucleon field operators, that faithfully reproduces the chiral Ward identities of QCD. The latter terms are not constrained by chiral symmetry and their parameters (low-energy constants, called LECs for brevity) must be determined from a fit to few-nucleon data. Following Weinberg, the power counting is performed on the level of the effective potential between two, three and four nucleons and a properly regularized Schrödinger-type equation is solved to generate the bound and scattering states. Life becomes much simpler if one goes to Euclidean time and formulates the theory on a space-time lattice of volume $(N a)^{3} \times\left(N_{t} a_{t}\right)$, with $a\left(a_{t}\right)$ the (temporal) lattice spacing and $N\left(N_{t}\right)$ the number of grid points in the space (time) direction. In fact, the lattice spacing $a$ serves as an $\mathrm{UV}$ regulator of the EFT as it implies a maximal momentum of $p_{\max } \sim \pi / a$. For a typical value of $a \simeq 2 \mathrm{fm}$, one has $p_{\max } \simeq 300 \mathrm{MeV}$, i.e. a very soft interaction. A further advantage of the lattice formulation is that it automatically takes into account all possible configurations allowed by symmetries, in particular, we can have up to four nucleons on one lattice site. Such configurations naturally lead to clustering, in fact, they require some special treatment so as not to produce overbinding of $\alpha$-like configurations. For that reason, the leading order (LO) interaction is smeared with a Gaussian, where the width parameter is adjusted to give the proper average S-wave NN effective range. Such smeared sources are also used frequently in lattice QCD calculations of hadron properties and dynamics [9]. This also means that the LO lattice EFT result should not be directly compared with a corresponding continuum calculation, where such effects are generated by higher order operators in the full potential. In the lattice approach, only the LO potential is summed up whereas all higher orders are treated in perturbation theory. This is consistent with the low momentum cutoff utilized. An important new development is the so-called nuclear lattice projection Monte Carlo (MC) technique [10]. We use a larger class of initial and final states than considered in our earlier work. I give some examples: For the calculation of ${ }^{4} \mathrm{He}$ we use an initial state with four nucleons, each at zero momentum. For the calculation of ${ }^{8} \mathrm{Be}$ we use the same initial state as for ${ }^{4} \mathrm{He}$, but then apply creation operators after the first time step to inject four more nucleons at zero momentum. The analogous process is done to extract four nucleons before the last step. This injection and extraction process of nucleons at
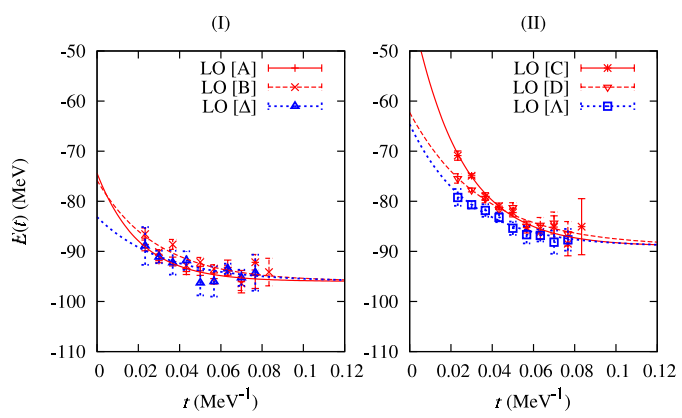

Figure 1. Lattice results for the ${ }^{12} \mathrm{C}$ spectrum at leading order. In Panel I results from three different initial states, A, B, and $\Delta$ are shown, each approaching the ground state energy. In Panel II results starting from three other initial states, $\mathrm{C}, \mathrm{D}$, and $\Lambda$ are displayed. These trace out an intermediate plateau at energy about $7 \mathrm{MeV}$ above the ground state. This is nothing but the celebrated Hoyle state.

zero momentum helps to eliminate directional biases caused by initial and final state momenta. To probe the structure of the ${ }^{12} \mathrm{C}$ states, we use not only delocalized initial and final states (configurations $\mathrm{A}, \mathrm{B}, \mathrm{C}, \mathrm{D}$ ) but also cluster-type wave functions (compact triangle $\Delta$, elongated triangle $\Lambda$ ). As seen in Fig. 1, while the configurations A,B and $\Delta$ approach the ground state energy, we do not find fast convergence to the ground state for configurations C,D and $\Lambda$, thus giving the structure of the first excited $0^{+}$state. The common thread connecting each of the initial states $\mathrm{C}, \mathrm{D}$, and $\Lambda$, is that each produces a state which has an extended or prolate geometry. The projection MC method is thus a powerful tool to unravel the structure of any considered nucleus and its excited states. 


\section{Fixing parameters and ground state results}

Next, we must fix the parameters (LECs) of our theory. At present, we work at next-to-next-toleading order (NNLO) in the chiral expansion, including strong and electromagnetic isospin breaking (the Coulomb force is included as described in [11]). We have 9 strong isospin-symmetric LECs. We fix these from the $\mathrm{S}$ - and P-waves in $n p$ scattering and the deuteron quadrupole moment, $Q_{d}$. The 2 LECs related to isospin-breaking are determined from a fit to the different $n p, p p$ and $n n$ scattering lengths, whereas the two LECs $C$ and $D$ of the leading three-nucleon force are fixed from the $n d$ doublet scattering length and the triton binding energy. The first non-trivial predictions are then the momentum dependence of the $p p{ }^{1} S_{0}$ partial wave, which agrees nicely with the Nijmegen PWA result [11] and the binding energy difference of the triton and ${ }^{3} \mathrm{He}$. We find $E\left({ }^{3} \mathrm{He}\right)-E\left({ }^{3} \mathrm{H}\right)=0.78(5) \mathrm{MeV}$ [12], in good agreement with the empirical value of $0.76 \mathrm{MeV}$.

The ground state energies for various nuclei are summarized in Tab. 1 . Consider first ${ }^{4} \mathrm{He},{ }^{8} \mathrm{Be}$ and ${ }^{12} \mathrm{C}$, which show some unique trends. We observe that the smeared LO contribution is already close to the empirical numbers for these nuclei, whereas the two-nucleon forces alone underbind at NNLO. The rescue comes from the three-nucleon forces at this order, which provide just the right amount of binding. These $3 \mathrm{~N}$ forces become more important as the atomic number $A$ increases, as is also observed in e.g. Green's function MC [13] or no-core shell model calculations [14]. The NNLO results for these nuclei are already quite satisfactory. Of course, higher orders and improved methods to extract the energies will have to be considered in the future. Work along these lines is in progress. The situation is somewhat different for ${ }^{16} \mathrm{O}$ (note that these are preliminary numbers which might still change a bit). Here, the smeared leading order leads to some overbinding, which is not completely corrected from the $3 \mathrm{~N}$ forces at NNLO. I will come back to this issue in Sec. 7.

Table 1. Ground state energies. LO denotes the smeared leading order, NNLO (XN) the contribution from $\mathrm{X}=2,3$ nucleon forces up-to-and-including NNLO and NNLO the total result at that order. Also, Exp. denotes the empirical values. The numbers for ${ }^{16} \mathrm{O}$ are preliminary. All energies in $\mathrm{MeV}$.

\begin{tabular}{|l|cccc|l|}
\hline nucleus & LO & NNLO (2N ) & NNLO (3N) & NNLO & Exp. \\
\hline${ }^{4} \mathrm{He}$ & $-28.0(3)$ & -24.9 & -3.4 & $-28.3(6)$ & -28.3 \\
\hline${ }^{8} \mathrm{Be}$ & $-55(2)$ & -48 & -7 & $-55(2)$ & -56.5 \\
\hline${ }^{12} \mathrm{C}$ & $-96(2)$ & -77 & -15 & $-92(3)$ & -92.2 \\
\hline${ }^{16} \mathrm{O}$ & $-147.5(5)$ & -121 & -20 & $-141.4(9)$ & -127.6 \\
\hline
\end{tabular}

\section{Spectrum and structure of carbon-12}

The spectrum of ${ }^{12} \mathrm{C}$ is one of the most challenging topics in nuclear theory. More precisely, the socalled Hoyle state, a $0^{+}$excitation about $7.7 \mathrm{MeV}$ above the ground state, has been an enigma to $a b$ initio calculations since it was predicted by Hoyle in 1954 [15] from a calculation of the abundance of carbon and oxygen through stellar fusion and experimentally verified at Caltech a few years later [16]. Before continuing, I have to define what is precisely meant by an ab initio calculation. In a first step, one fixes the underlying forces between two and three nucleons in few-body systems, i.e. bound and scattering observables with $A \leq 4$. Then, given the so determined forces, one solves the quantum $A$-body problem exactly, in this case using lattice Monte Carlo methods ${ }^{1}$. Any nuclear property for systems with $A \geq 5$ is then a prediction, the precision of the calculations can be systematically

\footnotetext{
${ }^{1}$ The interesting and successful fermion molecular dynamics calculation of Ref. [17] requires a fit to a broad range of nuclei to pin down the various model parameters, see also the contribution by Feldmeier and Neff to this conference [18].
} 
improved by going to higher order in the potential and improving the statistical and systematic errors of the MC signals. As with any new method, it is only accepted by the community if one is able to solve problems that could not be resolved before. That is why as a first application of the framework of nuclear lattice simulations we focused on the spectrum of ${ }^{12} \mathrm{C}$. In fact, based on standing waves properly projected onto zero total momentum as well as angular momentum and parity, the first ever ab initio calculation of the Hoyle state was reported in [19]. Since then, using the projection MC method, we have improved the calculations and gained further insight into the structure of the ground and excited state, as summarized in Tab. 2. The trends for the excited states are similar to the ground state

Table 2. Low-lying spectrum of ${ }^{12} \mathrm{C}$ at NNLO from $2 \mathrm{~N}$ and $3 \mathrm{~N}$ forces [10] in comparison to experiment.

\begin{tabular}{|c|cccc|}
\hline & $0_{1}^{+}$ & $2_{1}^{+}$ & $0_{2}^{+}$ & $2_{2}^{+}$ \\
\hline $2 \mathrm{~N}$ & -77 & -74 & -72 & -70 \\
$3 \mathrm{~N}$ & -15 & -15 & -13 & -13 \\
$2 \mathrm{~N}+3 \mathrm{~N}$ & $-92(3)$ & $-89(3)$ & $-85(3)$ & $-83(3)$ \\
\hline Exp. & -92.16 & -87.72 & -84.51 & $-82.6(1)[20]$ \\
& & & & $-82.13(11)[21]$ \\
\hline
\end{tabular}

- about $20 \%$ of binding from the $3 \mathrm{~N}$ forces is required to bring agreement between experiment and theory. Of particular interest besides the Hoyle state is the $2^{+}$excitation just $2 \mathrm{MeV}$ above it. This state can be interpreted as an excitation of the elongated triangle that features prominently in the structure of the Hoyle state. The predicted energy is in good agreement with recent measurements, see Tab. 2 (cf. also the group-theoretical anlysis of such cluster states in Ref. [22]). In Ref. [10], electromagnetic observables (charge radii, quadrupole moments and electromagnetic transitions among the even-parity states of ${ }^{12} \mathrm{C}$ ) were also given at LO - all these results are in reasonable agreement with experiment. However, the calculation of the higher order corrections turned out to be more complicated than the ones for the spectrum. This requires algorithmic improvements for a precise extraction of the corresponding matrix elements which are presently under investigation.

\section{Life on Earth - an accident?}

The Hoyle state has been heralded as a prime example of the anthropic principle ${ }^{2}$, which states that the parameters of the fundamental interactions take values that are compatible with the existence of carbon-based life and that the Universe is old enough for such life to have formed (for a recent review, see [24]). Computer simulations allow one to address the question of how sensitive the production of carbon and oxygen in hot stars is to the fundamental parameters of QCD+QED, the gauge field theories underlying the formation of atomic nuclei. This is one of the great strengths of the framework presented here, as I will outline in the following. In fact, the energy of the Hoyle state is just $379 \mathrm{keV}$ away from the $3 \alpha$ threshold, and the production rate of carbon in the $3 \alpha$ process scales with $\exp \left(-\Delta E / k_{B} T\right)$, with $\Delta E=E_{\text {Hoyle }}-3 E_{\alpha}, T$ is the stellar temperature and $k_{B}$ Boltzmann's constant. In earlier studies, it was shown that $\Delta E$ can be modified by $\pm 100 \mathrm{keV}$, so that one still has a sufficient production of carbon and oxygen [25]. This does not appear particularly fine-tuned, as variation of about $25 \%$ are consistent with carbon-oxygen based life. However, we have to translate this constraint on $\Delta E$ into constraints on the fundamental parameters of QCD+QED, here the average light quark mass $m_{q}$ and the fine-structure constant $\alpha_{\mathrm{QED}}$, see the recent works for more details [26, 27]. Note also that the strong coupling constant $\alpha_{S}$ is fixed from scale-setting through the nucleon mass, so

\footnotetext{
${ }^{2}$ See, however, Kragh [23] for a critical view of his issue.
} 
it can not be varied independently. In the following, we only consider small changes in $m_{q}$ and $\alpha_{\mathrm{QED}}$, so that $\left.\delta E_{i} \simeq\left(\partial E_{i} / \partial M_{\pi}\right)\right|_{M_{\pi}^{\mathrm{ph}}} \delta M_{\pi}+\left.\left(\partial E_{i} / \partial \alpha_{\mathrm{QED}}\right)\right|_{\alpha_{\mathrm{QED}}^{\mathrm{ph}}} \delta \alpha_{\mathrm{QED}}$, where the superscript 'ph' refers to the physical value and we use the Gell-Mann-Oakes-Renner relation $M_{\pi}^{2} \sim m_{q}$ to translate the quark mass dependence into the pion mass dependence. The knowledge of the pion mass dependence of the nuclear Hamiltonian is required to calculate the pertinent derivatives. In fact, consider any nuclear energy level or difference under small perturbations of the pion mass around its empirical value to leading order in the chiral expansion,

$$
\left.\frac{\partial E_{i}}{\partial M_{\pi}}\right|_{M_{\pi}^{\mathrm{ph}}}=\left.\frac{\partial E_{i}}{\partial \tilde{M}_{\pi}}\right|_{M_{\pi}^{\mathrm{ph}}}+\left.x_{1} \frac{\partial E_{i}}{\partial m_{N}}\right|_{m_{N}^{\mathrm{ph}}}+\left.x_{2} \frac{\partial E_{i}}{\partial g_{\pi N}}\right|_{g_{\pi N}^{\mathrm{ph}}}+\left.x_{3} \frac{\partial E_{i}}{\partial C_{0}}\right|_{C_{0}^{\mathrm{ph}}}+\left.x_{4} \frac{\partial E_{i}}{\partial C_{I}}\right|_{C_{I}^{\mathrm{ph}}},
$$

where $\tilde{M}_{\pi}$ refers to the explicit $M_{\pi}$-dependence from the pion propagator in the OPE contribution, and $x_{1}=\left.\left(\partial m_{N} / \partial M_{\pi}\right)\right|_{M_{\pi}^{\mathrm{ph}}}$ and $\left.x_{2}=\left(\partial g_{\pi N}\right) / \partial M_{\pi}\right)\left.\right|_{M_{\pi}^{\mathrm{ph}}}=\left.\left(1 / 2 F_{\pi}\right)\left(\partial g_{A} / \partial M_{\pi}\right)\right|_{M_{\pi}^{\mathrm{ph}}}-\left.\left(g_{A} / 2 F_{\pi}^{2}\right)\left(\partial F_{\pi} / \partial M_{\pi}\right)\right|_{M_{\pi}^{\mathrm{ph}}}$ are obtained from the quark mass dependence of the nucleon mass and the nucleon axial-vector coupling combined with the one of the pion decay constant utilizing lattice QCD data and chiral perturbation theory. The coefficients $x_{3}, x_{4}$ can be mapped uniquely on the quark mass dependence of the inverse nucleon-nucleon S-wave scattering lengths, denoted $\bar{A}_{s} \equiv \partial a_{s}^{-1} /\left.\partial M_{\pi}\right|_{M_{\pi}^{\mathrm{ph}}}$ and $\bar{A}_{t} \equiv \partial a_{t}^{-1} /\left.\partial M_{\pi}\right|_{M_{\pi}^{\mathrm{ph}}}$. These can be determined from chiral nuclear EFT, assuming some modeling based on resonance saturation [28]. The most recent analysis at NNLO gives $\bar{A}_{s}=0.29_{-0.23}^{+0.25}$, $\bar{A}_{t}=-0.18 \pm 0.10$ [29]. This introduces some amount of uncertainty, as discussed below. The various partial derivatives in Eq. (1) can be straightforwardly and precisely computed using the auxiliary field quantum Monte Carlo techniques (for details, see Ref. [27]). Independent of the precise values of $\bar{A}_{s}$ and $\bar{A}_{t}$, we find strong evidence that the physics of the $3 \alpha$-process is driven by $\alpha$-clustering, as speculated before (see e.g. [30]). The condition that $|\Delta E| \leq 100 \mathrm{keV}$ can be turned into an inequality that connects the quark mass change $\delta m_{q} / m_{q}$ with $\bar{A}_{s}$ and $\bar{A}_{t}$, i.e. $\left(c_{1} \bar{A}_{s}+c_{2} \bar{A}_{t}+c_{3}\right) \cdot\left(\delta m_{q} / m_{q}\right) \leq|\Delta E|$ (where the $c_{i}$ are calculated using our MC techniques), as depicted in Fig. 2, the so-called end-of-theworld plot. From that figure one sees that shifts in the light quark mass at the $\simeq 2-3 \%$ level are unlikely to be detrimental to the development of life. Tolerance against much larger changes cannot be ruled out at present, given the relatively limited knowledge of the quark mass dependence of the two-nucleon S-wave scattering parameters $\bar{A}_{s, t}$. Lattice QCD is expected to provide refined estimates of the scattering parameters in the future. Further, variations of the fine-structure constant $\alpha_{\mathrm{QED}}$ up to $\pm 2.5 \%$ are consistent with the requirement of sufficient carbon and oxygen production in stars.

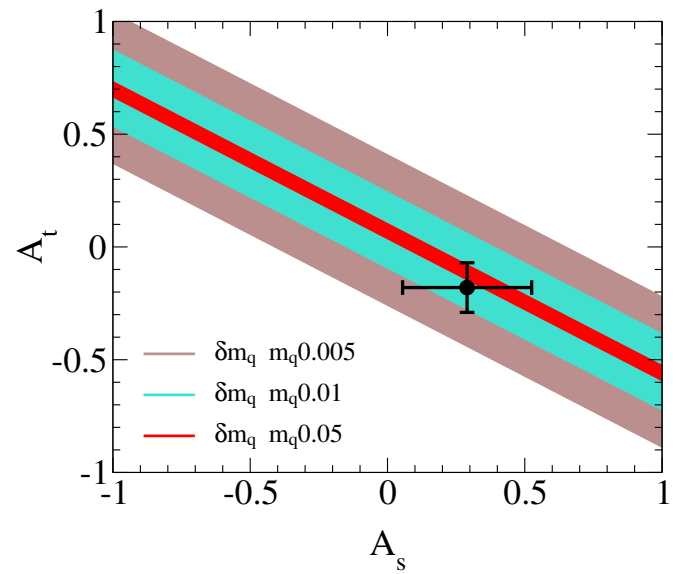

Figure 2. End-of-the-world plot. For relative quark mass changes of $0.5 \%$ (wide band), $1 \%$ (middle band) and $5 \%$ (inner most band), one can read off the allowed values for $\bar{A}_{s}$ and $\bar{A}_{t}$ (both varied within natural ranges from -1 to +1$)$, that are consistent with the requirements [25] for carbon-oxygen based life. The recent determination of $\bar{A}_{s}$ and $\bar{A}_{t}$ from Ref. [29] is depicted by the black cross. 


\section{Towards heavier nuclei}

Since the formalism outlined above does particularly well for $\alpha$-cluster states (note, however, that it is not restricted to such type of nuclei), it is natural to ask what happens if one extends these simulations to the next few $\alpha$-cluster nuclei, that are ${ }^{16} \mathrm{O},{ }^{20} \mathrm{Ne},{ }^{24} \mathrm{Mg}$, and ${ }^{28} \mathrm{Si}$. Here, I report some preliminary numbers for the ground state energies and the low-lying spectra of these nuclei. First, it is interesting to note that for this mass range the CPU time scales approximately as $A^{2}$ (whereas naively one would expect an $A^{3}$-scaling from the determinant calculation), so that the required computing time for ${ }^{28} \mathrm{Si}$ is only 5.4 times the one for the ${ }^{12} \mathrm{C}$ ground state. Second, we find that these heavier nuclei are overbound at NNLO, with the overbinding increasing with mass number, cf. Fig. 3. Let us consider the ${ }^{16} \mathrm{O}$ nucleus. Using the same action as described before leads to $E_{\text {g.s. }}\left({ }^{16} \mathrm{O}\right)=(-141 \pm 1) \mathrm{MeV}$, which is about $14 \mathrm{MeV}$ overbinding (for an earlier variational $\mathrm{MC}$ calculation based on non-chiral forces, see [31]). This effect can be traced back to the new $\alpha$-cluster geometries (the square and the tetrahedral structure, to be precise) that are possible with four or more alpha particles. These geometries generate too much attraction, similar to the four-nucleon configurations on one lattice site that required Gaussian smearing as discussed above. The simplest method to overcome this effect is to introduce a smeared four-nucleon operator, which is formally of higher order in the chiral expansion (for an earlier use of such type of operator, see e.g. Refs. [11, 12]). The strength of this operator can be fixed from the ground state energy of ${ }^{16} \mathrm{O}$. As can be seen from Fig. 3, this also leads to a good description of the ground state energies of all $\alpha$-cluster nuclei up to ${ }^{28} \mathrm{Si}$. Therefore, we believe that we have captured correctly the physics behind the binding energies of these nuclei. Clearly, these studies need to be backed by higher order calculations. In fact, we are presently extending the chiral potentials to next-to-next-to-next-to-leading order $\left(\mathrm{N}^{3} \mathrm{LO}\right)$. These exist in the continuum (see e.g. the $\mathrm{N}^{3} \mathrm{LO}$ three-nucleon forces in Refs. [32-34]) and just need to be adopted to the lattice notation.

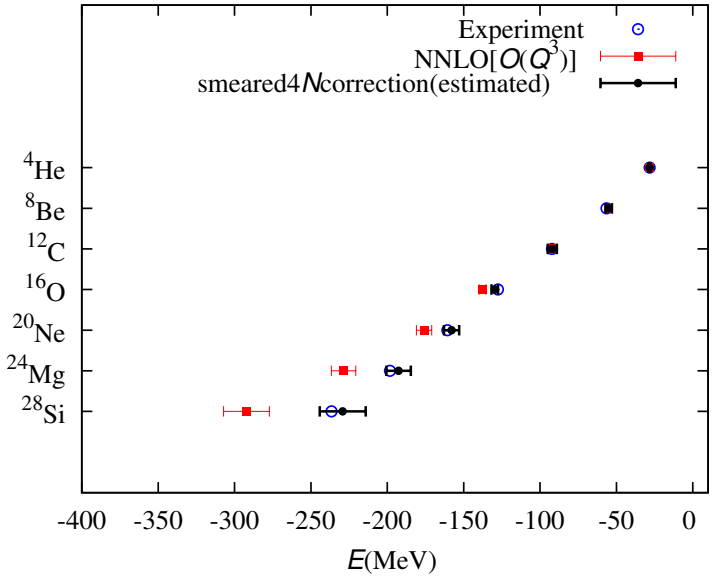

Figure 3. Ground state energies for $\alpha$-cluster nuclei at NNLO (red boxes) in comparison to the empirical numbers (blue open circles). Including a smeared four-nucleon contact interaction with its strength fixed from the ${ }^{16} \mathrm{O}$ ground state energy leads to much improved results (black circles). The numbers (in $\mathrm{MeV}$ ) are 158(5) [-160.6], 193(8) [-198.3] and 229(15) [-236.5] for ${ }^{20} \mathrm{Ne},{ }^{24} \mathrm{Mg}$, and ${ }^{28} \mathrm{Si}$, respectively, and the value of the experimental binding energy is given in the square brackets.

It is also interesting to study the low-lying spectrum of these nuclei. Consider again ${ }^{16} \mathrm{O}$. The tetrahedral structure is the dominant component in its ground state wave function with $J^{\pi}=0^{+}$and by its geometry it naturally leads to a $3^{-}$excitation. The square configuration is dominant in the first $0^{+}$excitation, that is located just below the $3^{+}$-state and has a $2^{+}$configuration as its lowest excitation. These configurations are exactly matching the experimentally found low-lying states in ${ }^{16} \mathrm{O}$ (see also [35]). In fact, we are presently performing a detailed analysis of this spectrum and the influence of the breaking of rotational symmetry of such configurations on the coarse lattices with $a \simeq 2 \mathrm{fm}$ used. 


\section{Summary and outlook}

To summarize, nuclear lattice simulations are a novel scheme to tackle the nuclear quantum manybody problem, firmly rooted in the so successful chiral effective field theory of the nuclear forces. To make further progress, methodological investigations in three directions are required. First, one has to address the topic of corrections beyond NNLO. Second, systematic studies of the lattice spacing dependence and the related issue of rotational symmetry breaking are required. Third, the hybrid MC algorithm has to be improved to get better signals for electromagnetic and weak operators. Work in all these directions is under way. Finally, I mention a few physics topics that are presently also under investigation:

- Reaction theory on the lattice. A general strategy for investigating capture reactions on the lattice as been outlined in Ref. [36]. The method consists of two major steps. First, projection MC is used to determine a multi-channel adiabatic lattice Hamiltonian for the participating nuclei. The second part is the calculation of the effective two-body capture reaction at finite volume using the adiabatic Hamiltonian. As a first application, the leading M1 contribution for $p(n, \gamma) d$ is worked out in [36]. See also Ref. [37] for an ab initio approach for fusion reactions using chiral forces.

- Topological volume corrections. Scattering processes on a lattice can be analyzed using Lüscher's method [38]. However, if the bound states (like nuclei) move in a periodic volume, additional topological volume corrections arise that are sensitive to the number and mass of the constituents [39] (and its extension to QFT in [40]). The importance of these corrections has been explicitely demonstrated in Ref. [41] for elastic scattering between a fermion and a bound dimer in the shallow binding limit (and is presently investigated in the study of $n d$ scattering [42]).

- Equation of state of neutron matter. The equation of state of neutron matter is currently a hot topic, see Steiner's talk at this conference. We are presently performing a NNLO analysis of the energy of neutron matter as a function of density, extending the work in Ref. [43], investigating also the pairing gap and possible P-wave pairing. For related works employing chiral EFT, see e.g. Refs. [44-46].

\section{Acknowledgments}

I would like to thank my colleagues from the NLEFT collaboration for sharing their insight into the topics presented here, especially Dean Lee and Timo Lähde. This work is supported in part by DFG and NSFC (SFB/TR 110 "Symmetries and the emergence of structure in QCD"), the HGF (Nuclear Astrophysics Virtual Institute, VH-VI-417), the BMBF (grant no. 05P12PDFTE) and by the EU (HadronPhysics3, grant no. 283286).

\section{References}

[1] U.-G. Meißner, Nucl. Phys. A 751, 149 (2005)

[2] S. Weinberg, Phys. Lett. B 251, 288 (1990)

[3] E. Epelbaum, H.-W. Hammer and U.-G. Meißner, Rev. Mod. Phys. 81, 1773 (2009)

[4] R. Machleidt and D. R. Entem, Phys. Rept. 503, 1 (2011)

[5] S. Bacca, Few Body Syst. 54, 903 (2013).

[6] S. Quaglioni, P. Navratil, G. Hupin, J. Langhammer, C. Romero-Redondo and R. Roth, Few Body Syst. 54, 877 (2013).

[7] B. Borasoy, E. Epelbaum, H. Krebs, D. Lee and U.-G. Meißner, Eur. Phys. J. A 31, 105 (2007) 
[8] D. Lee, Prog. Part. Nucl. Phys. 63, 117 (2009)

[9] C. Gattringer and C. B. Lang, Lect. Notes Phys. 788, 1 (2010).

[10] E. Epelbaum, H. Krebs, T. A. Lähde, D. Lee and U.-G. Meißner, Phys. Rev. Lett. 109, 252501 (2012)

[11] E. Epelbaum, H. Krebs, D. Lee and U.-G. Meißner, Eur. Phys. J. A 45, 335 (2010)

[12] E. Epelbaum, H. Krebs, D. Lee and U.-G. Meißner, Phys. Rev. Lett. 104 (2010) 142501

[13] S. C. Pieper, Riv. Nuovo Cim. 31, 709 (2008)

[14] R. Roth, S. Binder, K. Vobig, A. Calci, J. Langhammer and P. Navratil, Phys. Rev. Lett. 109, 052501 (2012)

[15] F. Hoyle, Astrophys. J. Suppl. 1, 121 (1954)

[16] C. W. Cook, W. A. Fowler, C. C. Lauritsen and T. Lauritsen, Phys. Rev. 107 (1957) 508.

[17] M. Chernykh, H. Feldmeier, T. Neff, P. von Neumann-Cosel and A. Richter, Phys. Rev. Lett. 98, 032501 (2007)

[18] H. Feldmeier and T. Neff, arXiv:1307.6449 [nucl-th]

[19] E. Epelbaum, H. Krebs, D. Lee and U.-G. Meißner, Phys. Rev. Lett. 106 (2011) 192501 [arXiv:1101.2547 [nucl-th]].

[20] M. Freer et al., Phys. Rev. C 80, 041303 (2009)

[21] W. R. Zimmerman et al., arXiv:1303.4326 [nucl-ex].

[22] R. Bijker and F. Iachello, Phys. Rev. C 61, 067305 (2000)

[23] H. Kragh, Arch. Hist. Exact Sci. 64, 721 (2010

[24] A. N. Schellekens, arXiv:1306.5083 [hep-ph].

[25] H. Schlattl, A. Heger, H. Oberhummer, T. Rauscher and A. Csoto, Astrophys. Space Sci. 291 (2004) 27

[26] E. Epelbaum, H. Krebs, T. A. Lähde, D. Lee and U.-G. Meißner, Phys. Rev. Lett. 110, 112502 (2013)

[27] E. Epelbaum, H. Krebs, T. A. Lähde, D. Lee and U.-G. Meißner, Eur. Phys. J. A 49:82 (2013)

[28] E. Epelbaum, U.-G. Meißner, W. Gloeckle and C. Elster, Phys. Rev. C 65, 044001 (2002)

[29] J. C. Berengut, E. Epelbaum, V. V. Flambaum, C. Hanhart, U.-G. Meißner, J. Nebreda and

J. R. Pelaez, Phys. Rev. D 87, 085018 (2013)

[30] S. Weinberg, Facing up, Harvard University Press (2001).

[31] S. C. Pieper, R. B. Wiringa and V. R. Pandharipande, Phys. Rev. C 46, 1741 (1992)

[32] S. Ishikawa and M. R. Robilotta, Phys. Rev. C 76, 014006 (2007)

[33] V. Bernard, E. Epelbaum, H. Krebs and U.-G. Meißner, Phys. Rev. C 77, 064004 (2008)

[34] V. Bernard, E. Epelbaum, H. Krebs and U.-G. Meißner, Phys. Rev. C 84, 054001 (2011)

[35] R. Bijker, J. Phys.: Conf. Ser. 380, 012003 (2012)

[36] G. Rupak and D. Lee, Phys. Rev. Lett. 111, 032502 (2013).

[37] P. Navratil and S. Quaglioni, Phys. Rev. Lett. 108, 042503 (2012)

[38] M. Lüscher, Nucl. Phys. B 354, 531 (1991).

[39] S. Bour, S. Koenig, D. Lee, H.-W. Hammer and U.-G. Meißner, Phys. Rev. D 84, 091503 (2011)

[40] Z. Davoudi and M. J. Savage, Phys. Rev. D 84, 114502 (2011)

[41] S. Bour, H.-W. Hammer, D. Lee and U.-G. Meißner, Phys. Rev. C 86, 034003 (2012)

[42] A. Rokash, E. Epelbaum, H. Krebs, D. Lee and U.-G. Meißner, arXiv:1308.3386 [nucl-th].

[43] E. Epelbaum, H. Krebs, D. Lee and U.-G. Meißner, Eur. Phys. J. A 40, 199 (2009)

[44] N. Kaiser, S. Fritsch and W. Weise, Nucl. Phys. A 697, 255 (2002) 
INPC 2013

[45] A. Lacour, J. A. Oller and U.-G. Meißner, Annals Phys. 326, 241 (2011)

[46] A. Gezerlis, I. Tews, E. Epelbaum, S. Gandolfi, K. Hebeler, A. Nogga and A. Schwenk, Phys. Rev. Lett. 111, 032501 (2013) 
